\title{
Cooperation Strategies for Two Co-located receivers, with no CSI at the transmitter
}

\author{
Amichai Sanderovich, Avi Steiner and Shlomo Shamai (Shitz) \\ Technion, Haifa, Israel \\ Email: \{amichi@tx,savi@tx,sshlomo@ee\}.technion.ac.il
}

\begin{abstract}
We study the case of a single transmitter, which communicates to two co-located users, through an independent block Rayleigh fading channel. The co-location nature of the users allows cooperation, which increases the overall achievable rate, from the transmitter to both users. The transmitter is ignorant of the fading coefficients, while receivers have access to perfect channel state information (CSI). This gives rise to the broadcast approach used by the transmitter. The broadcast approach facilitates reliable transmission rates adapted to the actual channel conditions, designed to maximize average throughput. With the broadcast approach, users can decode partly the total message, with almost any fading realization. The better the channel quality, the more layers that can be decoded. Such an approach is useful when considering average rates, rather than outage vs. rate (outage never occurs). The cooperation between the users is performed over the co-location channel, modeled as separated additive white Gaussian channels (AWGN), with an average power constraint, and limited or unlimited bandwidth. New achievable rates when combining cooperation with the broadcasting approach are presented, where through simple change of power allocation, substantial gains are demonstrated. We consider both amplify-and-forward (AF) and Wyner-Ziv compress-and-forward $(\mathrm{CF})$, as cooperation approaches, and also compare to decode-and-forward (DF). We extend these methods using the broadcast approach, and also to include separated processing of the layers, realized through multi-session cooperation. Further, novel closed form expressions for infinitely many multi-session AF and recursive expressions for the more complex multi-session CF are given. Numerical results for the various cooperation strategies demonstrate the efficiency of multi-session cooperation. Our results can be extended straightforwardly to a setting of a single transmitter sending common information for two users.
\end{abstract}

\section{INTRODUCTION}

In recent years, cooperation schemes constitute a significant research topic. Cooperating receivers is a particular example in this class [1],[2],[3] (among many others). Such cooperation can be achieved using compress-and-forward (CF) and amplify-and-forward (AF) techniques, which use lossy source coding techniques, so the cooperative receiver does not need to decode the message, or also decode-and-forward (DF), which requires decoding at the cooperative receiver [4]. Here, we deal with one transmitter that sends the same information to two co-located users, through independent, block Rayleigh fading channel [5]. We discuss both cases where the information is directed to a single destination and/or both co-located users are interested in decoding reliably the message. The Shannon capacity of this channel is zero, and usually one turns to rate versus outage probability [6] in such cases. When considering the average throughput or the delay as figures of merit, it may be beneficial to use the broadcast approach. The broadcast approach for a single-user facilitates reliable transmission rates adapted to the actual channel conditions, without providing any feedback from the receiver to the transmitter [7]. The single-user broadcasting approach hinges on the super-position coding for the broadcast channel, where every fading gain is associated with another user. In [8], a similar network setting is considered, with a single source transmitting to two co-located users, where a Wyner-Ziv (WZ) CF single session cooperation is studied, in a different setting. In this paper, we consider the case where the two receivers can cooperate between themselves over separated channels, so that they can improve each other's reception via source related techniques (AF and $\mathrm{CF}$ ). Since these users are co-located, the separated cooperative channels can use different frequency bands, so that unlike the channel from the transmitter, the probability of a multi-path non-line-of-sight channel is low, and the cooperation takes place over an additive white Gaussian (AWGN) channel, with a power constraint, and either limited or unlimited bandwidth. We present, in addition to the single session cooperation (referred to as naive $\mathrm{AF}$ and $\mathrm{CF}$ ) also multi-session cooperation scheme, like was done by [9] for the binary erasure channel. By combining the broadcasting approach with multi-session cooperation, we can enhance the efficiency of each session, by accounting for the information that was already decoded. Thus, the performance of the naive cooperation schemes is surpassed.

The rest of the paper is organized as follows. We give the problem setting, definitions and the used notations in sections II and II-A. Upper and lower bounds are stated for completeness in section III. Section IV deals with cooperation through the simpler amplify-and-forward, and section V improves the achievable rates of the previous section, by using the WynerZiv compression, namely the $\mathrm{CF}$.

\section{Problem Setting}

We study the problem of a single transmitter, which does not posses any knowledge of channel state information (CSI), communicating to a destination, whereas the destination and a co-located relay have access to perfect CSI. The perfect CSI is obtained through some preamble, and then the destination and the relay exchange their CSI, through the cooperation channels. The power used for this exchange is not included in the average power, since it is independent of the block 


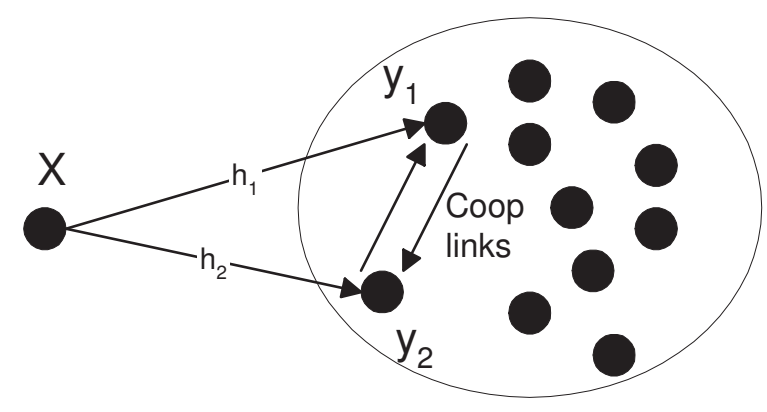

Fig. 1. Schematic diagram of a source transmitting to two co-located users, with multi-session cooperation.

length. The wireless network setting is illustrated in Figure 1. Our results for this problem setting are also valid for the case where a transmitter sends common information to two co-located users. See section VIII for more details.

\section{A. Channel Model}

Consider the following single-input multiple-output (SIMO) channel (we use boldfaced letters for vectors),

$$
\mathbf{y}_{i}=h_{i} \mathbf{x}_{s}+\mathbf{n}_{i} \quad, i=1,2
$$

where $\mathbf{y}_{i}$ is the received vector by user $i$, of length $L$, which is also the transmission block length. $\mathbf{x}_{s}$ is the transmitted vector, which satisfies $E\left|x_{s}\right|^{2} \leq P_{s}{ }^{1} . \mathbf{n}_{s}$ is the additive noise vector, with elements that are circularly symmetric Gaussian i.i.d with zero mean and unit variance, denoted $\mathcal{C N}(0,1)$, and $h_{i} \sim$ $\mathcal{C N}(0,1)(i=1,2)$ are two independent (scalar) block fading coefficients. Note that this results with the same average SNR for the two receivers, which is realistic due to their physical co-location. Without loss of generality we assume here that the desired destination is user $i=1$, unless explicitly stated otherwise. The cooperation channels between the users are modeled by AWGN channels as follows

$$
\begin{aligned}
& \mathbf{y}_{2,1}^{(k)}=\mathbf{x}_{1}^{(k)}+\mathbf{w}_{1}^{(k)} \\
& \mathbf{y}_{1,2}^{(k)}=\mathbf{x}_{2}^{(k)}+\mathbf{w}_{2}^{(k)}
\end{aligned}
$$

where $\mathbf{y}_{2,1}^{(k)}$ is the second user's received cooperation vector (of length $L$ ) from the destination $\left(i=1\right.$ ), on the $k^{\text {th }}$ cooperation link, and vise-versa for $\mathbf{y}_{1,2}^{(k)} \cdot \mathbf{x}_{i}^{(k)}$ is the cooperation signal from user $i$, on the $k^{t h}$ cooperation link, and $\mathbf{w}_{i} \sim \mathcal{C N}(0,1)$ is the additive noise. The power constraint here is $E \sum_{k=1}^{K}\left|x_{i}^{(k)}\right|^{2} \leq P_{r}(i=1,2)$, where for a single session cooperation $K=1$, and for a multi-session cooperation, $K>1$. The bandwidth expansion factor is also associated with $K$, that results from the multi-session cooperation, which is modeled by $K$-parallel cooperation channels. Notice that unlike the common reference to the relay channel, all these channels are separated, so no interference is introduced, unlike [8]. Also note that the users use half duplex communication equipment.

\footnotetext{
${ }^{1} E$ stands for the expectation operator.
}

Naturally, the link capacity of a single session narrow-band cooperation is given by

$$
C_{\text {coop }, N B}=\log \left(1+P_{r}\right) .
$$

In the limit of $K \rightarrow \infty$ with a power constraint for multisession cooperation, the cooperation link capacity is given by

$$
C_{\text {coop }, W B}=\int_{0}^{\infty} d R(s)=\int_{0}^{\infty} \rho(s) d s=P_{r},
$$

where $d R(s)$ is the fractional rate in session associated with parameter $s$, and $d R(s)=\log (1+\rho(s) d s)$. The fractional power at the $s^{t h}$ session is $\rho(s)$. The multi-session power constraint implies $\int_{0}^{\infty} \rho(s) d s=P_{r}$, which justifies the last equality in (22).

In view of the simplicity assumed by the naive AF strategy, we do not consider any bandwidth expansions, and use only the original signal bandwidth in a single session. However, all approaches may utilize a cooperation channel bandwidth expansion of the form $C_{c o o p, W B}$ (22) for improving the cooperation efficiency.

\section{UPPER AND LOWER BOUNDS}

In order to evaluate the benefit of cooperation among receivers in a fading channel following the model described in (1)-(2), we present two lower and upper bounds (LB and UB respectively) for both the broadcasting and outage techniques [7] that can be used by the transmitter. The bounds consist of outage and broadcasting average rates that are computed for a single user, assuming there are no available users for cooperation, for the lower bound and for a single receiver with two antennas and optimal processing for the upper bound, see [10]. All bounds are calculated assuming independent block Rayleigh fading. First, we address in the following the standard outage policy and the corresponding bounds read:

$$
\begin{aligned}
& R_{\text {outage }, L B}=\max _{u_{t h}}\left\{\left(1-F_{L B}\left(u_{t h}\right)\right) \log \left(1+u_{t h} P_{s}\right)\right\} \\
& R_{\text {outage }, U B}=\max _{u_{t h}}\left\{\left(1-F_{U B}\left(u_{t h}\right)\right) \log \left(1+u_{t h} P_{s}\right)\right\} \\
& R_{b s, L B}=e^{-1}-e^{-z_{0}^{l}}+2 E_{i}\left(z_{0}^{l}\right)-2 E_{i}(1) \\
& R_{b s, U B}=s_{1} e^{-z_{1}^{u}}-e^{-z_{1}^{u}}-3 E_{i}\left(z_{1}^{u}\right)- \\
&\left(z_{0}^{u} e^{-z_{0}^{u}}-e^{-z_{0}^{u}}-3 E_{i}\left(z_{0}^{u}\right)\right)
\end{aligned}
$$

where

$$
\begin{aligned}
F_{L B}(x) & =1-e^{-x} \\
F_{U B}(x) & =1-(1+x) e^{-x} \\
z_{0}^{l} & =2\left(1+\sqrt{1+4 P_{s}}\right)^{-1} \\
z_{0}^{u} & =I_{U B}^{-1}\left(P_{s}\right) \\
z_{1}^{u} & =I_{U B}^{-1}(0)
\end{aligned}
$$

where $I_{U B}(x)=\left(1+x-x^{2}\right) / x^{3}$. See [7] ${ }^{2}$.

$$
{ }^{2} E_{i}(x)=\int_{x}^{\infty} d t \frac{e^{-t}}{t}
$$




\section{AMPLIFY-AND-FORWARD COOPERATION}

For AF, we consider three types of cooperation schemes:

1) Naive amplify-and-forward $(n A F)$ - Each user operates standard AF using single session $(K=1)$, where the transmitter uses the optimal power allocation $I(x)$ (see [7]).

2) Separate preprocessing amplify-and-forward (spAF) The AF policy is used with the modification of removal of separately decoded layers prior to the forwarding.

3) Multi-session amplify-and-forward (msAF) - Multisession $\operatorname{AF}(K=\infty)$ repeatedly uses the separate preprocessing per session, and a total power constraint $P_{r}$ for all the cooperation sessions.

Clearly, when each scheme is operating at optimal setting, we have:

$$
R_{N A F} \leq R_{s p A F} \leq R_{m s A F},
$$

where $R_{n A F}, R_{s p A F}$ and $R_{m s A F}$ correspond to the average rates of the nAF, spAF and msAF strategies, respectively.

\section{A. Naive AF Cooperation}

In the naive AF strategy, the relaying user $(i=2)$ scales its input to the available transmit power $P_{r}$, and forwards the signal to the destination user $(i=1)$ using a single session $(K=1)$. The received signal at the destination after $\mathrm{AF}$ is then

$$
\mathbf{y}_{a}=\left(\mathbf{y}_{1}, \mathbf{y}_{1,2}^{(1)}\right)
$$

where $\frac{1}{1+\alpha} \mathbf{y}_{1,2}^{(1)}=\sqrt{\beta} \mathbf{x}_{s}+\widetilde{\mathbf{w}}_{2}$. The normalized noise vector $\widetilde{\mathbf{w}}_{2} \sim \mathcal{C} \mathcal{N}(0,1)$ hence the normalized signal gain after the scaling of user $i=2$ is $\beta=\frac{P_{r} s_{2}}{1+P_{s} s_{2}+P_{r}}$, where $s_{i}=\left|h_{i}\right|^{2}$. The achievable rate as a function of the channel fading gains is given by the following mutual information

$$
\begin{aligned}
\frac{1}{L} I\left(\mathbf{x}_{s} ; \mathbf{y}_{a} \mid\right. & \left.h_{1}, h_{2}\right)=\log \left(1+P_{s}\left(s_{1}+\beta\right)\right) \\
= & \log \left(1+P_{s}\left(s_{1}+\frac{P_{r} s_{2}}{1+P_{s} s_{2}+P_{r}}\right)\right) .
\end{aligned}
$$

Therefore the continuous broadcasting equivalent fading parameter is $s_{a}=s_{1}+\beta$, rather then $s_{1}$, if no cooperation was used. This requires the derivation of the CDF of $s_{a}$, [10]. For a Rayleigh fading the CDF of $s_{a}$ is explicitly given by

$$
\begin{gathered}
F_{s_{a}}(x)=1- \\
\begin{cases}1 & x \leq 0 \\
e^{-\frac{\left(1+P_{r}\right) x}{P_{r}-P_{s} x}}+\int_{0}^{\frac{\left(1+P_{r}\right) x}{P_{r}-P_{s} x}} d u e^{-u-x+\frac{P_{r} u}{1+P_{s} u+P_{r}}} & 0 \leq x<\frac{P_{r}}{P_{s}} \\
\int_{0}^{\infty} d u e^{-u-x+\frac{P_{r} u}{1+P_{s} u+P_{r}}} & x \geq \frac{P_{r}}{P_{s}} .\end{cases}
\end{gathered}
$$

We get the following proposition:

Proposition 4.1: Using naive AF, at the co-located users, and broadcasting with the matching power allocation at the transmitter, the following average rate is achievable:

$$
R_{n A F}=\int_{x_{0}}^{x_{1}} d x\left[\frac{2\left(1-F_{s_{a}}(x)\right)}{x}+\frac{\left(1-F_{s_{a}}(x)\right) f_{s_{a}}^{\prime}(x)}{f_{s_{a}}(x)}\right]
$$

where $x_{0}$ and $x_{1}$ are determined from the boundary conditions $I_{r}\left(x_{0}\right)=P_{s}$ and $I_{r}\left(x_{1}\right)=0$, where

$$
I_{r}(x) \triangleq \frac{1-F_{s_{a}}(x)-x f_{s_{a}}(x)}{f_{s_{a}}(x) x^{2}}
$$

and $F_{s_{a}}(x), f_{s_{a}}(x), f_{s_{a}}^{\prime}(x)$ are the CDF of $s_{b}$, its first and its second derivatives, respectively. For the Rayleigh channel, with transmitter power $P_{s}$ and cooperation power $P_{r}, F_{s_{a}}(x)$ is (12)

Proof: See [10].The proof follows from the optimization of the average rate over the power allocations, through calculus of variations. The resulting power allocation used by the transmitter is denoted by $I_{r}(x)$.

\section{B. AF with Separate Preprocessing}

Let us write the received signal at the $i^{t h}$ user by

$$
\boldsymbol{y}_{i}=h_{i}\left(\boldsymbol{x}_{s, D_{i}}+\boldsymbol{x}_{s, I\left(s_{i}\right)}\right)+\boldsymbol{n}_{i},
$$

where $\boldsymbol{x}_{s, D_{i}}$ is the part of the signal successfully and separately decoded by user $i$. The residual interference signal is then denoted $\boldsymbol{x}_{s, I\left(s_{i}\right)}$, which includes coded layers which could not get separately decoded. As [10], define the power allocation according to $I\left(s_{i}\right) \triangleq \mathrm{E}\left|x_{s, I\left(s_{i}\right)}\right|^{2}$. Since the users have full CSI, they can agree on the minimum fading $j=$ $\operatorname{argmin}_{i}\left\{s_{i}\right\}$ for determining the commonly decoded signal $\boldsymbol{x}_{s, D_{j}}$ (notice that it depends only on the two fading realizations, not on the AWGN). For example, when $s_{1} \geq s_{2}$, after removing commonly decoded layers up to $s_{2}$ (at both users), the residual interference power is given by $I\left(s_{2}\right)$. The signals to be scaled and amplified are then given by: $\boldsymbol{y}_{i, I}=h_{i} \boldsymbol{x}_{s, I\left(s_{j}\right)}+\boldsymbol{n}_{i}$.

Following the same lines of the naive AF (11), the equivalent fading gain at user $i=1$, after amplifying and forwarding $y_{2, I\left(s_{j}\right)}$, is

$$
s_{a}=s_{1}+\frac{P_{r} s_{2}}{1+s_{2} I\left(s_{j}\right)+P_{r}} .
$$

Proposition 4.2: In an AF with separate preprocessing cooperation strategy, with a single cooperation session $K=1$ (power $P_{r}$ ) and power allocation $I(x)$ (power constraint $\left.I\left(x_{0}\right)=P_{s}\right)$ at the transmitter, the rate

$$
R_{s p A F}=\int_{x_{0}}^{\infty}\left(1-F_{s_{a}}(x)\right) \frac{-x I^{\prime}(x)}{1+x I(x)} d x
$$

is achievable. The CDF $F_{s_{a}}(x)$ is calculated from (16) and given in [10].

Proof: See [10].

Note that the cooperation power can be increased by $\frac{1}{1-P_{b s}}$, where $P_{b s}$ is the probability that the destination will successfully decode all layers without cooperation, so that power 
can be saved. Observe that since (16) includes $I(x)$, so does $\left.F_{s_{a}}(x)\right)$. This turns the optimization problem of the power allocation to be a difficult one, unlike the naive AF.

\section{Multi-Session AF with Separate Preprocessing}

In this scheme, we repeatedly use the technique of reducing commonly decoded information and then forwarding. The total power allocation available for all sessions is still $P_{r}$, where unlike previous schemes, here $K=\infty$. We find the average rate for unlimited number of sessions, assuming only an overall power constraint for all sessions $P_{r}$. It should be emphasized that the multi-session is performed over parallel channels (for example, OFDM), in such way that the source transmission is block-wise continuous. So that we use wideband cooperation channel here.

In the case of unlimited sessions, the scalar equivalent fading gain can be derived for a given broadcasting power allocation $I(s)$.

Proposition 4.3: In a multi-session $A F(K=\infty$, cooperation power constraint $P_{r}$ ) with separate preprocessing cooperation strategy, the highest decodable layer is associated with an equivalent fading gain determined by $s_{a}^{*}$, (assuming $\left.s_{1}>s_{2}\right)$. To compute it, start with $s_{b}^{*}$, which is the solution of the following equation,

$$
\int_{s_{2}}^{s_{b}^{*}} \frac{s_{1}}{\left(s_{1}+s_{2}-\sigma\right)^{2}}\left[1+s_{1} I(\sigma)\right] d \sigma=P_{r}
$$

and by using $s_{b}^{*}$,

$$
s_{a}^{*}=s_{1}+s_{2} \frac{Z\left(s_{b}^{*}\right)}{1+Z\left(s_{b}^{*}\right)} .
$$

where

$$
Z(s)=\int_{s_{2}}^{s} \frac{1+s_{1} I(\sigma)}{\left(1+s_{2} I(\sigma)\right)} \frac{s_{1}}{\left(s_{1}+s_{2}-\sigma\right)} d \sigma .
$$

Since the agents are equivalent, the averaged rate received by the destination is $R_{m s A F}=\frac{1}{2} \mathrm{E}\left[\log _{2}\left(1+s_{a}^{*} P_{s}\right)+\log _{2}(1+\right.$ $\left.\left.s_{b}^{*} P_{s}\right)\right]$.

Proof: See [10]. From the equivalent fading gain (19) and the statistics of $s_{1}, s_{2}$, a CDF for $s_{a}^{*}$ can be computed, from which the average achievable rate can be obtained.

In order to improve performance, the above approach uses power allocation also for the cooperation channels, not only for the transmitter, which is denoted by $\rho(s)$ (that is, $\mathrm{E}\left|x_{i}^{(k)}\right|^{2}=$ $\int_{s_{b}^{(k-1)}}^{s^{(k)}} \rho(s) d s$, where $s_{b}^{(k)}$ is equivalent fading after the $k^{t h}$ session). The optimal power allocation for the cooperation sessions as a function of the achieved gain $s$, at the weaker user $i=2$, given $I(x)$ and $s_{1}, s_{2}$ is

$$
\rho(s)=\left(1+s_{1} I(s)\right) \frac{s_{1}}{\left(s_{1}+s_{2}-s\right)^{2}} .
$$

Since the cooperation is performed over parallel channels, with infinitesimal power allocated per channel, the capacity of this wide-band cooperation link is

$$
C_{\text {coop }}=\int_{0}^{\infty} d R(s)=\int_{0}^{\infty} \rho(s) d s=P_{r} .
$$

Notice that the previous AF schemes (sections IV-A and IVB), can not effectively use such capacity increase $\left(P_{r}>\right.$ $\log \left(1+P_{r}\right)$ ), because they do not encode the transmission prior to forwarding. In any case, the above approach outperforms all spectral extension techniques for a wide-band naive AF, since here each session reduces the effect of the additive noise that is being forwarded.

The exact solution for optimal $I(x)$ is an open problem. So for the numerical results we use $I(s)$ corresponding to optimal broadcasting in presence of optimal joint decoding. This selection is demonstrated (see Section VII) to be a good one, particularly for high $P_{s}$ and $P_{r}$, as such conditions allow approximation of optimal performance with multi-session AF cooperation.

\section{COMPRESS-AND-FORWARD COOPERATION}

In this section we consider compress-and-forward cooperation. Both users are capable of performing compressing and forwarding (CF) of a quantized signal to one another. The compression here relies on the well known Wyner-Ziv [11] compression using side information at the decoder, which in this case, is the received channel output. Similar to the AF, here too, we consider three ways of implementing the basic cooperation.

\section{A. Naive CF Cooperation}

Consider the channel model in (1)-(2). The signal to be sent to the destination, which the compressed version of the received signal at the relay $\hat{\boldsymbol{y}}_{2}$, is given by

$$
\hat{\boldsymbol{y}}_{2}=\boldsymbol{y}_{2}+\boldsymbol{n}_{c}=h_{2} \boldsymbol{x}_{s}+\boldsymbol{n}_{2}+\boldsymbol{n}_{c},
$$

where $n_{c} \sim \mathcal{C N}\left(0, \sigma^{2}\right)$ is the compression noise, which is independent of $y_{2}$. Then the maximal achievable rate to the destination, is given by

$$
\begin{aligned}
& R_{C F, 1}\left(h_{1}, h_{2}\right)=\frac{1}{L} I\left(\mathbf{x}_{s} ; \mathbf{y}_{1}, \hat{\boldsymbol{y}}_{2} \mid h_{1}, h_{2}\right) \\
& \text { s.t. } I\left(\mathbf{y}_{2} ; \hat{\boldsymbol{y}}_{2} \mid h_{2}\right)-I\left(\mathbf{y}_{1} ; \hat{\boldsymbol{y}}_{2} \mid h_{1}, h_{2}\right) \leq L C_{\text {coop }}
\end{aligned}
$$

where $R_{C F, 1}\left(h_{1}, h_{2}\right)$ is maximized when the constraint is met with equality. The constraint $C_{\text {coop }}$ represents the cooperation link capacity. According to the channel model there is unlimited bandwidth and a $P_{r}$ power limitation. We mention two cases for the naive CF:

1) Narrow-band naive CF: $K=1$, and therefore $C_{\text {coop }}=$ $\log \left(1+P_{r}\right)$.

2) Wide-band naive CF: $K=\infty$ and therefore $C_{\text {coop }}=P_{r}$.

Only the first one is considered, as it already significantly outperforms the AF schemes. The resulting quantization noise is $\mathrm{E}\left|n_{c}\right|^{2}=\sigma^{2}$ which for the narrow-band naive $\mathrm{CF}$ equals $\sigma_{N B}^{2}=\frac{1+s_{1} P_{s}+s_{2} P_{s}}{P_{r}\left(1+s_{1} P_{s}\right)}$. This leads to the next proposition:

Proposition 5.1: In a Narrow-band Naive compress-andforward strategy, given $s_{1}, s_{2}$, and $P_{r}, P_{s}$, as defined in section II-A (with $K=1$ ), the highest decodable layer in user $i$ is associated with an equivalent fading gain determined by

$$
s_{n C F, i}=s_{i}+\frac{s_{3-i}\left(1+s_{i} P_{s}\right) P_{r}}{\left(1+P_{r}\right)\left(1+s_{i} P_{s}\right)+s_{3-i} P_{s}} .
$$


The distribution of $s_{n C F, i}$ for a Rayleigh fading $F_{s_{n C F}}(u)$ can be expressed in a closed expression, as done for the AF, which is given in [10], for the sake of brevity.

\section{B. CF with separate preprocessing}

We repeat what was done in section IV-B, also for the $\mathrm{CF}$. That is, instead of first performing $\mathrm{CF}$, both users can separately decode, and then use $\mathrm{CF}$, which is now performed with better side information, in the form of the commonly decoded information. For consistency, assume that $s_{1}>s_{2}$, and then replace $P_{s}$ from $\sigma_{N B}^{2}$ by $I\left(s_{2}\right)$, the equivalent signal to noise ratio at $i=1$, after the first iteration is now written by (16) and (25):

$$
s_{1}^{(1)}=s_{1}+\frac{s_{2} P_{r}\left(1+s_{1} I\left(s_{2}\right)\right)}{\left(1+P_{r}\right)\left(1+s_{1} I\left(s_{2}\right)\right)+s_{2} I\left(s_{2}\right)} .
$$

\section{Multiple sessions with $C F$ and separate preprocessing}

As was done for the amplify-and-forward, can be repeated for the compress-and-forward processing. Define the auxiliaries variables $(i=1,2) \hat{\boldsymbol{y}}_{i}^{(k)}=\boldsymbol{y}_{i}+\boldsymbol{n}_{c, i}^{(k)}$, where $n_{c, i}^{(k)}$ is independent with $y_{i}, y_{3-i}$, as the compression of $\boldsymbol{y}_{i}$, which is decoded at the other user $(3-i)$ during the $k^{t h}$ session. Since we use multiple WZ compressions, each with increased side information, we refer the reader to [12], for the successively refinable Wyner-Ziv. Here, we deal with the case where the message that is transmitted in each session has better side information than the previous session, since more layers are decoded. Further, the second session can use the information sent by all the previous sessions, in order to improve performance. Since the power that is used by each session is a control parameter, rather than a fixed parameter, as in the multi-session AF, the use of an auxiliary compression variable that is transmitted during a session, but decoded only at the next session (due to the better side information, declared as $V$ in [12]) is inefficient. Next, using [12], the following Markov chains are defined, where unlike [12], we are interested in independent averaged distortion, rather than plain averaged quadratic distortion.

$$
\begin{aligned}
& y_{2}-x_{s}-y_{1}-\hat{y}_{1}^{(k)}-\hat{y}_{1}^{(k-1)}-\cdots-\hat{y}_{1}^{(1)} \\
& y_{1}-x_{s}-y_{2}-\hat{y}_{2}^{(k)}-\hat{y}_{2}^{(k-1)}-\cdots-\hat{y}_{2}^{(1)}
\end{aligned}
$$

The resulting equivalent fading gains after every iteration of the multi-session cooperation are stated in the following proposition.

Proposition 5.2: The achievable rate in the multi-session with separate preprocessing and successively refinable WZ is given in a recursive form for the $k^{\text {th }}$ session, for user $i=1$, for $s_{1}, s_{2}, \operatorname{Pr}, I(x)$ :

$$
R_{C F, i=1}^{(k)}=E_{s_{1}^{(k)}} \log \left(1+s_{1}^{(k)} P_{s}\right)
$$

where

$$
s_{i}^{(k)}=s_{i}+\frac{s_{3-i}}{1+\left(\sigma_{2}^{(k)}\right)^{2}}
$$

and

$$
\begin{gathered}
\left(\sigma_{i}^{(k)}\right)^{2}=\left(\sigma_{i}^{(k-1)}\right)^{2}\left(1+s_{i} I\left(s^{(k-1)}\right)+s_{3-i} I\left(s^{(k-1)}\right)\right) \\
\left\{\left(1+s_{3-i} I\left(s^{(k-1)}\right)\right)\left[1+\delta_{i}^{(k)}\left(1+\left(\sigma_{i}^{(k-1)}\right)^{2}\right)\right]\right. \\
\left.+s_{i} I\left(s^{(k-1)}\right)\left(1+\delta_{i}^{(k)}\right)\right\}^{-1}
\end{gathered}
$$

for $i=1,2$, and where $\mathrm{E}\left|x_{i}^{(k)}\right|^{2}=\delta_{i}^{(k)}$ and $s^{(k-1)} \triangleq$ $\min _{i=1,2}\left\{s_{i}^{(k-1)}\right\}$. Proof: See [10].

\section{DECODE Forward COOPERATION}

For a fair comparison of DF cooperation to the other multisession techniques we consider both wide-band cooperation, where $C_{\text {coop }}=P_{r}$ (22), and narrow-band cooperation (corresponding to the single session relaying techniques), where the cooperation link capacity is only $C_{\text {coop }}=\log \left(1+P_{r}\right)$. The DF strategy for our setting can be described as follows. The source performs continuous broadcasting, and two copies of the transmitted signal are received at the destination and the relay users, as described by the channel model (1)-(2). Recalling that the destination is denoted by user $i=1$, then for $s_{1} \geq s_{2}$ the destination user can decode at least as many layers as the relay user. Hence there is place for DF cooperation only when $s_{1}<s_{2}$, as in this case the relaying user can decode more layers than the destination. The additional layers decoded by the relay (for $s \in\left(s_{1}, s_{2}\right]$ ) are encoded by the relay and then forwarded, constrained by the capacity of the cooperation channel. Thus for $P_{r}>>P_{s}$, which is a practically unlimited cooperation channel, all additional information may be sent to destination and the strongest user upper bound is obtained. Denote the decodable rate associated with a fading gain $s$ by $R(s)$, where $R(s)=\int_{0}^{s} d u \frac{-I^{\prime}(u) u}{1+I(u) u}$ [7]. Say that before cooperation starts user $i$ decodes $R\left(s_{i}\right)$. Hence for the pair $\left(s_{1}, s_{2}\right)$, the achievable broadcasting rate is given by

$R_{D F}\left(s_{1}, s_{2}\right)= \begin{cases}\min \left\{R\left(s_{1}\right)+C_{\text {coop }}, R\left(s_{2}\right)\right\} & s_{2}>s_{1} \\ R\left(s_{1}\right) & \text { otherwise }\end{cases}$

The optimal broadcasting power distribution maximizes the average rate, and the optimization problem is stated as follows,

$$
\begin{gathered}
\bar{R}_{D F}=\max _{\rho(s) \geq 0, \text { s.t. } \int_{0}^{\infty} d s \rho(s) \leq P_{s}} E_{s_{1}, s_{2}} R_{D F}\left(s_{1}, s_{2}\right) \\
=\max _{\rho(s) \geq 0, \text { s.t. } \int_{0}^{\infty} \rho(s) d s \leq P_{s}} \\
\left\{\int_{0}^{\infty} d s_{2} \int_{0}^{s_{2}} d s_{1} f\left(s_{1}\right) f\left(s_{2}\right) \min \left\{R\left(s_{1}\right)+C_{\text {coop }}, R\left(s_{2}\right)\right\}\right. \\
\left.+\int_{0}^{\infty} d s_{2} \int_{s_{2}}^{\infty} d s_{1} f\left(s_{1}\right) f\left(s_{2}\right) R\left(s_{1}\right)\right\} .
\end{gathered}
$$

Finding the optimal power allocation seems intractable analytically, however $\bar{R}_{D F}$ could be computed for sub-optimal power distributions, such as the strongest user $I_{\text {sel,opt }}(s)$, or for the no cooperation $I_{S U, o p t}(s)$, and also for $I_{J o i n t, o p t}(s)$. These are defined and demonstrated in section VII. 


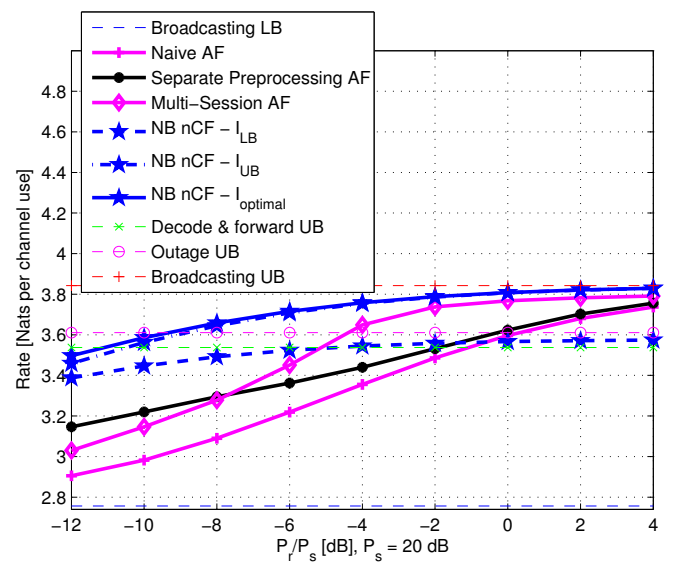

Fig. 2. Broadcast: Average rates of Naive AF, AF with separate preprocessing, multi sessions $\mathrm{AF}$ and narrow-band naive $\mathrm{CF}$ (with $I(x)$ taken from (7) for $I_{L B},(8)$ for $I_{U B}$ and the optimal, related to (25), respectively) compared to upper and lower bounds, as function of the channels quality ratio $\frac{P_{r}}{P_{S}}$, when $P s=20 \mathrm{~dB}$.

\section{NUMERICAL RESULTS}

In this section, we compare the various methods, where we use narrow band for all the schemes, besides the multi-session. Figure 2 shows a comparison between $\mathrm{nAF}$, spAF,msAF, and narrow-band $\mathrm{nCF}$, as function of the channels quality ratio $P_{r} / P_{s}$. Where we used the power allocation of nAF in spAF and $I(s)=\frac{1}{s^{3}}+\frac{1}{s^{2}}-\frac{1}{s}$ (optimal for joint) in msAF, both are suboptimal. As may be noticed from the figure, the lower $P_{r} / P_{s}$, the higher the rate gains of spAF, over the nAF. For $P_{s}=20 \mathrm{~dB}$ and $P_{r} / P_{s} \geq 0$, both approaches outperform the upper bound for the achievable rate when using the outage approach. In moderate to high $P_{s}$ and $P_{r}$, the multi-session AF approximates the upper bound of the achievable rate when using broadcasting. The naive $\mathrm{CF}$, again, outperforms all other approaches, and approximates the broadcasting upper bound even on a wider range of $P_{r}$ values. The figure also plots an upper bound on the performance of DF, which for $\operatorname{Pr}>P s-1.5 d B$, are inferior to all the others. The figure also demonstrates the implications of using sub-optimal power allocation for broadcasting in the narrow-band naive $\mathrm{CF}$ approach. It may be noticed that the difference between the optimal and UB optimal allocation is rather small, unlike the LB optimal allocation. This assures us that the evaluated msAF, is not far from the optimal msAF. We suspect that separate processing and multi-session $\mathrm{CF}$ could close further the gap to the broadcasting UB.

\section{DISCUSSION AND CONCLUSION}

We have considered several cooperation strategies for transmission to co-located users. The original data is intended to one of the users and, in the network setting examined, a colocated user receives another copy of the original signal and cooperates with the destination user to improve decoding at the destination. As the transmitter has no access to CSI, the broadcast approach is used along with various cooperation strategies. We have presented and examined the naive AF, along with improved versions, denoted by separate preprocessing $\mathrm{AF}$ and multi-session AF. In separate preprocessing AF, the users individually decode as many layers as they can, subtract the common information and forward a scaled version of the residual signal. In a multi-session AF approach, this is repeated infinitely. We gave an explicit formulation for all these techniques. Another cooperation approach considered was $\mathrm{CF}$, which was also improved through preprocessing. Using multisession CF brought notions such as successive refinable WZ coding. Explicit expression was derived for the naive $\mathrm{CF}$ and numerical results showed that naive $\mathrm{CF}$ outperforms all other AF approaches (for which we computed average rates). The multi-session AF with a sub-optimal broadcasting power allocation also presented good results, although only over a wide-band cooperation channel. The results here are also valid, through straightforward transformation, for the case when a single source sends common information to two cooperating users. The impressive performance of the $\mathrm{CF}$ scheme, and the improvement of multi-session over single session in the $\mathrm{AF}$, indicate the possible performance benefits of using multisession $\mathrm{CF}$ (msCF). However, the optimization problem for $\mathrm{msCF}$ seems to be un-solvable analytically.

\section{ACKNOWLEDGMENT}

This research has been supported by the REMON consortium.

\section{REFERENCES}

[1] C. T. K. Ng, I. Maric, A. J. Goldsmith, S. Shamai, and R. D. Yates, "Iterative and one-shot conferencing in relay channels," in Proc. of IEEE Information Theory Workshop (ITW2006), Punta del Este, Uruguay, Mar.

[2] A. Sanderovich, S. Shamai, Y. Steinberg, and G. Kramer, "Communication via decentralized processing," Submitted to IEEE trans. on info. Theory, Nov. 2005, see also [13].

[3] R. Dabora and S. D. Servetto, "Broadcast channels with cooperating decoders," IEEE Trans. Inform. Theory, vol. 52, no. 2, pp. 5438-5454, Dec 2006.

[4] G. Kramer, M. Gastpar, and P. Gupta, "Cooperative strategies and capacity theorems for relay networks," IEEE Trans. Inform. Theory, vol. 51, no. 9, pp. 3037-3063, Sept. 2005.

[5] R. S. Blum, "Distributed detection for diversity reception of fading signals in noise," IEEE Trans. Inform. Theory, vol. 45, no. 1, pp. 158164, 1999.

[6] E. Biglieri, J. Proakis, and S. Shamai, "Fading channels: Informationtheoretic and communications aspects," IEEE Trans. Inform. Theory, vol. 44, no. 6, pp. 2619-2692, Oct. 1998.

[7] S. Shamai (Shitz) and A. Steiner, "A broadcast approach for a single user slowly fading MIMO channel," IEEE Trans. on Info, Theory, vol. 49, no. 10 , pp. 2617-2635, Oct. 2003.

[8] M. Katz and S. Shamai(Shitz), "Relaying protocols for two co-located users," to appear in IEEE Trans. on Inform. Theory, vol. 52, no. 6, 2006.

[9] S. C. Draper, B. J. Frey, and F. R. Kschischang, "Interactive decoding of a broadcast message," in In Proc. Allerton Conf. Commun., Contr., Computing, IL, Oct.

[10] A. Steiner, A. Sanderovich, and S. Shamai, "Broadcast cooperation strategies for two co-located users," Submitted to IEEE trans. on info. Theory.

[11] A. D. Wyner and J. Ziv, "The rate-distortion function for source coding with side information at the decoder," IEEE Trans. Inform. Theory, vol. 22, no. 1, pp. 1-10, January 1976

[12] Y. Steinberg and N. Merhav, "On successive refinement for the WynerZiv problem," IEEE Trans. on Info. Theory, vol. 50, no. 8, pp. 16361654, Aug. 2004.

[13] A. Sanderovich, S. Shamai, Y. Steinberg, and G. Kramer, "Communication via decentralized processing," in Proc. of IEEE Int. Symp. Info. Theory (ISIT2005), Adelaide, Australia, Sep. 2005, pp. 1201-1205. 